\title{
Lysimeter Leaching Study of Cyantraniliprole
}

\author{
V. N. Kolupaeva ${ }^{1}$, A. A. Belik ${ }^{1,2}$, A. A. Kokoreva ${ }^{2}$ \\ ${ }^{1}$ Russian Scientific-Research Institute of Phytopathology \\ Bolshiye Vyazemy, Moscow region, Russia \\ v.kolupaeva@vniif.ru \\ ${ }^{2}$ Lomonosov Moscow State University \\ Moscow, Russia \\ belikalexandra@gmail.com; kaa@penreg.ru
}

\section{Extended Abstract}

Modern agriculture is impossible without the use of plant protection products. In addition to the expected effect of protection from pests, diseases and weeds, the use of pesticides often has an adverse effect on the environment and nontarget organisms. In particular, numerous studies have revealed the discovery of pesticide residues in groundwater around the world. For regulation purpose, in order to assess pesticide environmental risks in Russia, the experimental data obtained in the EU is used. The climate in Russia is colder with significantly higher water percolation than in Europe, which leads to a higher risk of pesticide migration to groundwater. This is also facilitated by the movement of plant protection products with preferential water flows through macropores and cracks.

The purpose of the research was to study the migration of the insecticide cyantraniliprole in the soil profile. Cyantraniliprole is a moderately persistence $\left(\mathrm{DT}_{50}=34.4\right.$ days $)$ medium-mobility $\left(\mathrm{K}_{\mathrm{oc}}=241\right)$ substance.

The experiment was carried out at the lysimeters of the Soil Research Station of Moscow State University from June 2015 to May 2017. The soil of lysimeter is soddy-podzolic silt loam. The insecticide was used at the recommended $(0.4 \mathrm{~kg}$ $\mathrm{ha}^{-1}$ ) and tenfold rates in June 2015 and then in June 2016. Water leachate from lysimeter was collected every week. Soil samples were collected every $5 \mathrm{~cm}$ till depth of $50 \mathrm{~cm}$ in spring and autumn. Cyantraniliprole was analysed by HPLC. Detection limits of the analytical method was $0.5 \mu \mathrm{g} / \mathrm{L}$ and $2.5 \mu \mathrm{g} / \mathrm{kg}$ for water and soil respectively.

The average annual air temperature in the years of the experiment was close to the average long-term values. The amount of precipitation in 2016 exceeded the mean annual value by $90 \mathrm{~mm}$, and in the summer period of 2016 - by 121 $\mathrm{mm}$. In 2015, average precipitation values for the year and for the seasons were close to the average annual parameters. However, in the summer of 2015 heavy showers with a daily rainfall rate exceeding a quarter of the monthly norm were observed.

The maximum depth of migration of cyananthraniliprole in the soil profile was $35 \mathrm{~cm}$ in October 2015 and $40 \mathrm{~cm}$ in October 2016. Despite the fact that cyanantraniliprole is a medium-persistence, one year after the second treatment (in May 2017), a rather large concentration of the pesticide (near 35\% of applied rate) was in the soil, which distributed to a depth of $30 \mathrm{~cm}$, with a maximum in the upper 5 -cm layer.

Cyantraniliprole was found in the leachate of lysimeter water 2 weeks after first application in both lysimeters (with the recommended and tenfold doses), the pesticide concentrations were 0.8 and $1.5 \mu \mathrm{g} / \mathrm{L}$ respectively. This was facilitated by the precipitation of several showers with rainfall more than $20 \mathrm{~mm}$. This indicates a high mobility of the pesticide in this soil and a large influence of rainfall on the rapid arrival of the pesticide beyond the soil profile.

Cyantraniliprole was found in most of the analyzed water samples. The pesticide in the water leachate wasn't found in 2015 in $22 \%$ of the samples at the recommended dose and in $6 \%$ of the samples at a tenfold dose. In 2016 and 2017 , cyantraniliprole was detected in all the selected aqueous samples. 